\title{
eNeuro
}

Research Article: New Research / Disorders of the Nervous System

\section{NPRL2 Inhibition of mTORC1 Controls Sodium Channel Expression and Brain Amino Acid Homeostasis}

https://doi.org/10.1523/ENEURO.0317-21.2022

Cite as: eNeuro 2022; 10.1523/ENEURO.0317-21.2022

Received: 26 July 2021

Revised: 7 February 2022

Accepted: 8 February 2022

This Early Release article has been peer-reviewed and accepted, but has not been through the composition and copyediting processes. The final version may differ slightly in style or formatting and will contain links to any extended data.

Alerts: Sign up at www.eneuro.org/alerts to receive customized email alerts when the fully formatted version of this article is published.

Copyright (@) 2022 Hui et al.

This is an open-access article distributed under the terms of the Creative Commons Attribution 4.0 International license, which permits unrestricted use, distribution and reproduction in any medium provided that the original work is properly attributed. 
1

2

3

4

NPRL2 Inhibition of mTORC1 Controls Sodium Channel Expression and Brain Amino Acid Homeostasis. Jeremy B. Hui, Jose Cesar Hernandez Silva, Mari Carmen Pelaez, Myriam Sévigny, Janani Priya Venkatasubramani, Quentin Plumereau, Mohamed Chahine, Christophe D. Proulx, Chantelle F. Sephton, Paul A. Dutchak

Department of Neuroscience and Psychiatry, CERVO Brain Research Centre, Université Laval, Quebec City, Quebec, G1J 2G3, Canada

\section{Abbreviated Title: NPRL2 Controls Neuronal Activity}

Corresponding Author: paul.dutchak.1@ulaval.ca

Number of pages: ( 1 title page +23 text pages $=24$ pages $)$

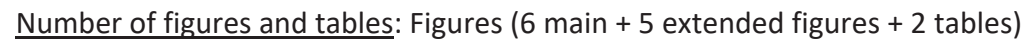

Number of words: Abstract (195), Introduction (694), Discussion (1110)

Conflict of Interest Statement: The authors declare that the research was conducted in the absence of any commercial or financial relationships that could be construed as a potential conflict of interest.

Acknowledgments: This research was supported by the Fonds de Recherche du Québec Santé Junior-1 (to C.D.P., C.F.S., P.A.D.), the Natural Sciences and Engineering Research Council of Canada (RGPIN-201706131 to C.D.P.; RGPIN-2020-06376 and DGECR-2020-00060 to C. F. S.; RGPIN-2018-06227 and DGECR2018-00093 to P.A.D.), the Canadian Institutes of Health Research (PJT169117 to C.D.P.; MOP-111072 and MOP-130373 to M.C.), institutional funding from the CERVO Brain Research Centre and a TSC Alliance Biorepository Seed Grants (to P.A.D.), the Natural Sciences and Engineering Research Council of Canada URSA scholarship (to J.B.H.) and the Mexican National Council for Science and Technology (CONACYT; No 471999 to J.C.H.S.) and a Québec-Mexico Doctoral research scholarship from the Fonds de Recherche en Santé du Québec No 257680 (to J.C.H.S.). We thank Dr. Benjamin Tu at the University of Texas Southwestern Medical Center for kindly providing the Nprl2 floxed mice, CERVO neuron culture platform members, and Ashley St. Pierre at the Analytical Facility for Bioactive Molecules, The Hospital for Sick Children, Toronto Canada for assistance with mass spectrometry analysis. 
Abstract:

Genetic mutations in nitrogen permease regulator-like 2 (NPRL2) are associated with a wide spectrum of familial focal epilepsies, autism, and sudden unexpected death of epileptics (SUDEP), but the mechanisms by which NPRL2 contributes to these effects are not well known. NPRL2 is a requisite subunit of the Gap Activity TOward Rags 1 (GATOR1) complex, which functions as a negative regulator of mammalian Target Of Rapamycin Complex 1 (mTORC1) kinase when intracellular amino acids are low. Here we show that loss of NPRL2 expression in mouse excitatory glutamatergic neurons causes seizures prior to death, consistent with SUDEP in humans with epilepsy. Additionally, the absence of NPRL2 expression increases mTORC1-dependent signal transduction and significantly alters amino acid homeostasis in the brain. Loss of NPRL2 reduces dendritic branching and increases the strength of electrically stimulated action potentials in neurons. The increased action potential strength is consistent with elevated expression of epilepsy-linked, voltage-gated sodium channels in the NPRL2-deficient brain. Targeted deletion of NPRL2 in primary neurons increases the expression of sodium channel $\operatorname{Scn} 1 A$, whereas treatment with the pharmacological mTORC1 inhibitor called rapamycin prevents $\operatorname{Scn} 1 A$ upregulation. These studies demonstrate a novel role of NPRL2 and mTORC1 signaling in the regulation of sodium channels, which can contribute to seizures and early lethality.

Significance Statement: NPRL2 is a requisite subunit of the epilepsy-linked GATOR1 complex that functions as a negative regulator of mTORC1 kinase when intracellular amino acids are limited. Here we report the generation and characterization of a new neurological model of GATOR1-dependent mTORopathy, caused by the loss of NPRL2 function in glutamatergic neurons. Loss of NPRL2 increases mTORC1 signal transduction, significantly alters amino acid homeostasis in the brain, and causes SUDEP. In addition, loss of NPRL2 increases the strength of electrically stimulated action potentials and the expression of epilepsy-linked sodium channels. These data reveal an unanticipated link between 
intracellular amino acid signaling by NPRL2 and a novel mTORC1-dependent regulation of sodiumchannel expression in epilepsy.

\section{Introduction:}

Nitrogen permease regulator-like 2 (NPRL2) is a requisite subunit of Gap Activity TOward Rags 1 (GATOR1), an evolutionarily conserved complex that is comprised of three proteins called NPRL2, NPRL3, and DEPDC5 (DEP Domain Containing 5). Recent genomic studies have identified mutations in all subunits of GATOR1 that collectively represent approximately $10 \%$ of the epileptic and autistic population (Baldassari et al., 2019; Dibbens et al., 2013; Ricos et al., 2016). Mutations in the DEPDC5 have also been suggested to be a causal factor for sudden unexpected death of epileptics (SUDEP) in humans (Nascimento et al., 2015). Individuals with GATOR1 mutations can present with focal cortical dysplasia, with defects in cortical lamination and the presence of dysmorphic neurons (Cen et al., 2017; Kabat and Król, 2012; Scerri et al., 2015; Weckhuysen et al., 2016). These malformations have been found to occur through a two-hit genetic mechanism (Ribierre et al., 2018), confounding the specific GATOR1-dependent mechanisms that contribute to neurological dysfunction.

GATOR1 functions as a negative regulator of mTORC1 (mammalian Target Of Rapamycin Complex 1), a dynamic protein kinase that controls cellular growth, protein translation, and metabolic processes in cells (Bar-Peled et al., 2013; Liu and Sabatini, 2020). mTORC1 activity is controlled by distinct upstream regulatory protein complexes comprised of GATOR1 and TSC1/2, which function as GTPase activating proteins toward small GTP-binding proteins on the lysosomal surface called RAGs and Rheb, respectively (Efeyan et al., 2013; Meikle et al., 2007; Sancak et al., 2010). The interaction of mTORC1 with GTPbound RAG and Rheb contributes to the activation of its kinase function (Laplante and Sabatini, 2009). Mutations in either GATOR1 or TSC1/2 are generally classified as mTORopathies, which present with overlapping neurological hallmarks, including seizures and autism spectrum disorders (Griffith and 
Wong, 2018; Talos et al., 2012). These similarities suggest an important role for strict mTORC1 regulation in the control of brain function (Curatolo et al., 2015; Specchio et al., 2020).

When intracellular amino acids are limited, GATOR1 represses mTORC1-dependent signaling and reduces the anabolic consumption of amino acids (Bar-Peled et al., 2013; Bourdeau Julien et al., 2018; Shen et al., 2019). Previous loss-of-function studies have shown that NPRL2 is necessary to maintain intracellular amino acids homeostasis by regulating the expression of genes important for metabolism of amino acids, including glutamine and glutamate (Chen et al., 2017; Dutchak et al., 2018; Dutchak et al., 2015). While intracellular glutamate functions as a building block for general protein synthesis, it can also contribute to cellular energy metabolism through mitochondrial anapleurosis or function as an excitatory neurotransmitter of glutamatergic neurons (Zhou and Danbolt, 2014).

Within the brain, glutamine contributes to the synthesis of both excitatory and inhibitory neurotransmitters in glutamatergic or GABAergic neurons, respectively. The metabolism of these neurotransmitters is highly inter-dependent within distinct cell populations, comprising the glutamineglutamate/GABA cycle to adequately supply neurotransmitters within the central nervous system. This cycle describes the shuttling of glutamine from astrocytes to glutamatergic or GABAergic neurons for neurotransmission, and subsequent re-uptake in astrocytes for glutamine regeneration (Schousboe et al., 2013). Alterations in this cycle are frequently observed in epileptic patients and have been associated with defects in astrocyte glutamine synthesis, or changes in glutaminase and glutamate dehydrogenase (Eid et al., 2016). These metabolic links to epilepsy prompted us to investigate the role of NPRL2 in glutamatergic neurons, which utilize glutamate as an excitatory neurotransmitter. While the contribution of other GATOR1 proteins, namely DEPDC5, have been explored in the GABAergic neurons in zebrafish (Swaminathan et al., 2018), the role of NPRL2 in glutamatergic neurons and its contribution to the mammalian brain has not been investigated. 
Here we report the generation and characterization of a new neurological model of GATOR1-dependent mTORopathy, caused by the loss of NPRL2 function in glutamatergic neurons. Loss of NPRL2 is sufficient to increase mTORC1 signaling despite an intact TSC1/2 complex, significantly alter amino acid homeostasis in the brain and cause seizures prior to early death. In addition, loss of NPRL2 increases the strength of electrically stimulated action potentials and the expression of epilepsy-linked sodium channels (Kaplan et al., 2016; Lakhan et al., 2009). These data reveal an unanticipated link between intracellular amino acid signaling through GATOR1 and a novel mTORC1-dependent regulation of sodium channel expression.

\section{Materials and Methods:}

\section{Animal Experiments}

NPRL2 nKO mice were generated by crossing $\mathrm{Npr} / 2^{\text {loxP/+ }}$ mice previously described (Dutchak et al., 2015), with VgluT2-ires-Cre females mice (Vong et al., 2011) to generate glutamatergic neuron-specific heterozygous animals. The expression of Cre-recombinase in these mice is regulated by an internal ribosomal entry site that was created using a knock-in strategy targeting the VgluT2 gene. Heterozygous animals were backcrossed to C57/B6 for at least 3 generations, with subsequent crosses with $\mathrm{Nprl} 2^{\text {loxp/+ }}$ and $\mathrm{Npr} / 2^{\text {IoxP/loxP }}$ animals, resulting in control mice $\left(\mathrm{Npr} / 2^{\text {loxP/+ }}\right.$ and $\left.\mathrm{Npr} / 2^{\text {loxP/loxP }}\right)$, NPRL2 nHET mice $\left(\mathrm{Nprl} 2^{\text {loxP/+}} ;\right.$ VgluT2-ires-Cre) and NPRL2 nKO mice $\left(\mathrm{Nprl} 2^{\text {loxP/loxP }} ;\right.$ VgluT2-ires-Cre) that were used for these studies. Genotypes of all animals were determined by PCR, using the following primers: Nprl2 (forward) 5'-CTCAGGTTCTACGCAGTGACTTC-3', Nprl2 (reverse) 5'-CATGGCGCTGTCTGGATCC-3', Np/2 (knockout) 5'CAGGCTTCATACTTCTACCCTC-3', VgluT2 (forward) 5'-AAGAAGGTGCGCAAGACG-3', VgluT2 (Cre-reverse) 5'-ACACCGGCCTTATTCCAAG-3', VgluT2 (wt-reverse) 5'-CTGCCACAGATTGCACTTGA-3'. Video recordings were captured using a Sony FDR-AX53 camcorder, with the mother present in the cage. All animal experiments were approved by the Université Laval Committee on Ethics and Animal Research. 


\section{Immunohistochemistry}

Tissue sections were prepared from P16 animals that were perfused with $4 \%$ (wt/vol) formaldehyde and immersion fixed in $4 \%$ formaldehyde for 16 hours. Floating sections were immunostained with primary antibodies overnight at $4^{\circ} \mathrm{C}$ using a 1:500 antibody dilution shown in Table 1. Alexa Fluor-conjugated secondary antibodies (Invitrogen) were incubated for 2 hours at room temperature prior to washing and mounting. Three animals per each genotype were analyzed using confocal microscope.

\section{Western Blot Analysis}

Tissue were homogenized in lysis buffer containing of $150 \mathrm{mM}$ sodium chloride, $50 \mathrm{mM}$ sodium fluoride, $100 \mu \mathrm{M}$ sodium orthovanadate $(\mathrm{pH}$ 10.0), $50 \mathrm{mM}$ sodium pyrophosphate tetrabasic, $10 \mathrm{mM} \beta-$ glycerophosphate, 5 mM EDTA, 5 mM EGTA, 10 mM HEPES ( $p H$ 7.4) and 0.5\% Triton X-100 supplemented with complete anti-protease cocktail (Roche). Lysates were pelleted by centrifugation. Cleared lysates, representing the soluble fraction, and the insoluble membrane fractions were boiled in 1X Laemmli sample buffer and separated on SDS-PAGE for Western blotting using antibodies listed in Table 1.

\section{Golgi Staining}

NPRL2 nKO and littermate controls were used for Golgi staining, using the manufacturer's protocol of the FD Rapid GolgiStain ${ }^{\mathrm{TM}}$ kit (FD Neurotechnologies, Inc.). 10 cortical neurons (layers IV-V) from three independent animals per genotype were traced and analyzed using Neurolucida 360 (MBF Biosciences, Williston, VT). Sholl analysis was performed starting at $10 \mu \mathrm{m}$ from the soma with $5 \mu \mathrm{m}$ intervals as previously described (Sephton et al., 2014).

\section{Slice Preparation for Electrophysiology}


Mice were first anesthetized with isoflurane. The brain was then quickly dissected and placed in the cutting chamber filled with an ice-cold NMDG-artificial cerebrospinal fluid (aCSF) solution containing (in mM): $1.25 \mathrm{NaH2PO}, 2.5 \mathrm{KCl}, 10 \mathrm{MgCl}$, 20 HEPES, $0.5 \mathrm{CaCl} 2,24 \mathrm{NaHCO}$, 8 D-glucose, 5 L-ascorbate, 3 Na-Pyruvate, 2 Thiourea, 93 NMDG (osmolarity adjusted with sucrose to 300-310 mOsmol/l) and $\mathrm{pH}$ adjusted to 7.4 with $\mathrm{HCl} 10 \mathrm{~N}$. Coronal slices $(250 \mu \mathrm{m})$ were then cut with a vibratome (VT2000; Leica) to obtain complete sections containing lateral habenula. Slices were placed in a $32^{\circ} \mathrm{C}$ oxygenated NMDGaCSF solution for 10 minutes before incubation for one hour at room temperature in HEPES-aCSF solution (in mM): $1.25 \mathrm{NaH} 2 \mathrm{PO} 4,2.5 \mathrm{KCl}, 10 \mathrm{MgCl} 2,20 \mathrm{HEPES}, 0.5 \mathrm{CaCl}$, 24 NaHCO3, 2.5 D-glucose, 5Lascorbate, $1 \mathrm{Na}$-Pyruvate, 2 Thiourea, $92 \mathrm{NaCl}, 20$ Sucrose (osmolarity adjusted to 300-310mOsmol/I at $\mathrm{pH}$ 7.4) and finally transferred into a recording chamber on the stage of an upright microscope (Zeiss) where it was perfused at a rate of 3-4 ml/min with artificial cerebrospinal solution (aCSF in $\mathrm{mM}$ ): 120 $\mathrm{NaCl}, 5 \mathrm{HEPES}, 2.5 \mathrm{KCl}, 1.2 \mathrm{NaH} 2 \mathrm{P0}$, $2 \mathrm{MgCl}$, $2 \mathrm{CaCl}$, 2.5 glucose, $24 \mathrm{NaHCO}, 7.5$ sucrose. The aCSF in the perfusion chamber was kept at $32^{\circ} \mathrm{C}$. All solutions were aerated with $95 \% \mathrm{O} 2$ and $5 \% \mathrm{CO} 2$. (Ting et al,2018).

\section{Whole cell patch clamp recordings}

A water immersion x60 objective and a video camera (Zeiss, Germany) were used to visualize neurons in LHb. Whole-cell patch clamp recordings were performed under current clamp with an Axopatch 200B amplifier (Molecular Devices, San Jose, CA) using borosilicate patch electrodes (3-7 M $\Omega$ resistance). Pipettes were filled with an intracellular patch solution containing (in mmol/l): $130 \mathrm{~K}$-Gluconate, $5 \mathrm{KCl}$, 10 HEPES, $2.5 \mathrm{MgCl}_{2}, 4 \mathrm{Na}_{2}$-ATP, $0.4 \mathrm{Na}_{3}$-GTP, 10 Na-phosphocreatine, 0.6 EGTA and 0.2\% Biocytin (pH 7.35). Signals were filtered at $5 \mathrm{kHz}$ using a Digidata $1500 \mathrm{~A}$ data acquisition interface (Molecular Devices, San Jose, CA) and acquired using pClamp 10.6 software (Molecular Devices, San Jose, CA). Pipette and cell capacitances were fully compensated. The pH and osmolarity were adjusted to 7.3 and 285-290 mOsm/L, respectively. Data were analyzed offline using clampfit 10.6 software. Neurons were 
selected randomly from the LHb of prepared slices. 1-2 minutes after obtaining whole cell configuration, the resting membrane potential (RMP) was recorded in current clamp mode right after whole cell configuration had been obtained. Rheobase current was assessed by applying depolarizing current steps (from -20 to $100 \mathrm{pA}, 5 \mathrm{pA}$ increments and $1 \mathrm{~s}$ duration) and it was calculated as the minimum current needed to elicit the action potential. To examine evoked firing properties, depolarizing current steps (20 to +100 pA, 20 pA increments and 300 ms duration) were applied to the cells. Action potentials (APs) generated during this period were counted and we obtained the number of spikes and frequency of firing.

\section{Metabolite Analysis}

The cortex of NPRL2 nKO and littermate controls were harvested from living P16 animals showing no physical signs of stress, by cervical dislocation, rapid dissection, and snap freezing in liquid nitrogen. Samples were stored at $-80^{\circ} \mathrm{C}$ until processing. One hemisphere was subjected to metabolite extraction using the AbsoluteIDQ ${ }^{\circledR}$ p180 kit (Biocrates Life Sciences), using the manufacturer's protocol. Samples were analyzed on a SCIEX 5500 QTRAP by The Analytical Facility for Bioactive Molecules, The Hospital for Sick Children, Toronto, Canada.

\section{Primary Neuron Cultures}

Primary cortical neurons were prepared from P0-P1 Nprl2f/fl mice using previously described methods (Nault and De Koninck, 2010). Dissociated neurons were grown in neurobasal medium supplemented with B27 (Thermo fisher), L-Glutamine (Fisher Scientific) and penicillin/streptomycin (Thermofisher). On DIV4 cultures were treated with $5 \mu \mathrm{M}$ AraC (Sigma) and either control or CRE expressing lentivirus. On DIV7 cells were treated with 10nM rapamycin or DMSO (vehicle) for 24 hours and harvested in TRIzol (Thermofisher) for RNA isolation.

\section{Quantitative RT-PCR Analysis}


Total RNA from tissues or cells was extracted using TRIzol ${ }^{\circledR}$ (Invitrogen), following the manufacturers protocols. Primer were selected to span exon-exon junctions where possible. RNA extracts were treated with DNase (Roche) and the High Cap cDNA Reverse Transcription kit (Applied Biosystems) was used for cDNA synthesis. Quantitative RT-PCR reactions contained $25 \mathrm{ng}$ cDNA, $150 \mathrm{nM}$ of each primer pair, and 5 uL SYBR GreenER (Invitrogen). All reactions were performed in triplicate on the QuantStudio $^{\text {TM }} 5$ Real-Time PCR system (Applied Biosystems). Relative mRNA levels were calculated using the comparative threshold cycle method using U36B4 as the internal control. qRT-PCR primer sequences used in this study are listed in Table 2.

\section{Statistical Analysis}

Statistical analysis was performed by two-tailed Student's t test using Microsoft Excel 2016. A p-value of $<0.05$ was considered significant.

\section{Results:}

\section{Loss of NPRL2 in Glutamatergic Neurons Causes Seizures and Early Lethality}

To determine the function of NPRL2 in glutamatergic neurons, we crossed our genetically engineered Nprl2-floxed mice with VgluT2-ires-Cre females mice to generate Nprl2 ${ }^{\text {loxP/loxP }} ;$ VgluT2-ires-Cre ${ }^{+/ W T}$ (NPRL2 nKO) mice (Dutchak et al., 2015; Vong et al., 2011). We selected this Cre-driver because it is widely expressed early in developing glutamatergic neurons, permitting us to investigate early onset epileptic phenotypes. To verify NPRL2 deletion, we performed western blot analysis targeting NPRL2 and other protein subunits of GATOR1, NPRL3 and DEPDC5, in cerebral protein extracts from WT and NPRL2 nKO animals. NPRL2 expression was reduced by $50 \%$ in the NPRL2 nKO compared to control, indicating a targeted knockout of NPRL2 in the VGLUT2 neuron population (Fig. 1A, 1B). Consistent with previous observations, loss of NPRL2 did not alter NPRL3 or DEPDC5 expression in the NPRL2 nKO (Dutchak et al., 2015). Animals were born at the expected Mendelian ratios; however, NPRL2 nKO mice did not live 
beyond 20 days after birth (Fig. 1C). We measured the body mass of NPRL2 nKO mice at P16 and found a small but significant reduction in both males and females compared to littermate controls (Fig. 1D). To investigate the cause of death, we performed 24-hour video recordings of control and NPRL2 nKO littermates, with their mother present. Control mice were unremarkable, whereas all NPRL2 nKO mice began a series of seizures approximately 3 hours before the start of the 12-hour light cycle on the day of their death. Over the next 2 to 4 hours, both tonic and tonic-clonic seizures were observed, lasting $1.6 \pm$ 0.6 minutes and $5.6 \pm 1.4$ minutes, respectively (Extended Data Fig. 1-1). NPRL2 nKO pups died following a tonic-clonic seizure lasting more than 5 minutes. These observations show that NPRL2 function in glutamatergic neurons is essential for viability, despite the presence of an intact TSC1/2 complex.

\section{NPRL2 contributes to dendritic branching and soma size of neurons}

Previous studies have shown that deletion of the GATOR1 subunit called DEPDC5, using a pan-neuronal Cre driver, reduces dendritic branching of neurons (Yuskaitis et al., 2018). To determine if the loss of NPRL2 alters dendritic branching in our model, we performed Golgi staining and Sholl analysis of layer IV-V cortical neurons in the NPRL2 nKO and littermate controls at P16 (Fig. 2A). We observed a significant decrease in the number of intersections and cumulative area of NPRL2 nKO neurons, compared to control (Fig. 2B, 2C). We also performed 3D reconstruction of the cell bodies to determine if the loss of NPRL2 increased the soma size, consistent with established pro-growth effects of mTORC1. We observed the soma of NPRL2 nKO neurons were 1.5-fold larger compared to control (Fig. 2D). No gross morphological differences were observed in the NPRL2 nKO brain compared to controls at P16 (data not shown). These data support NPRL2 as a critical regulator of dendritic branching and neuron size in vivo.

\section{Loss of NPRL2 alters intrinsic electrophysiological properties in neurons}


Mutations in NPRL2 are associated with familial forms of epilepsy (Baldassari et al., 2019; Ricos et al. 2016; Weckhuysen et al., 2016). To determine if the loss of NPRL2 alters the intrinsic passive and active neuronal electrophysiological properties, we prepared acute brain slices encompassing the lateral habenula (LHb), which contain exclusively VGLUT2-positive neurons, from NPRL2 nKO and control littermates. To determine neuronal firing properties, evoked firing activity was evaluated by injecting depolarizing current steps. We observed no significant difference in the number of action potentials (AP) (Fig. 3A, 3B), or instantaneous firing frequency (Fig. 3C) elicited by progressive depolarizing current steps. However, the electrophysiological properties of the first AP evoked by each depolarizing step showed significantly greater AP amplitude (Fig. 3D, 3E), and reduced rise time (Fig. 3F) in the NPRL2 nKO neurons compared to littermate controls. No significant difference was observed in the first AP decay slope, resting membrane potential, or rheobase current (Fig. 3G, 3H, 3I). Consistent with the larger size of the NPRL2 nKO neurons (Fig. 2D), a significant increase in capacitance was observed in these cells compared to control (Fig. 3J). These data suggest that loss of NPRL2 increases sodium-dependent depolarization step of the AP.

\section{NPRL2 controls mTORC1 signal transduction and neurometabolic homeostasis}

NPRL2 is an essential protein subunit of the GATOR1 complex, which functions as a negative regulator of mTORC1 when intracellular amino acids are limited. To determine if NPRL2 is necessary to repress mTORC1 activity our model, we performed immunohistochemistry and confocal microscopy on sections from NPRL2 nKO and control animals at P16, stained with phosphorylated ribosomal protein S6 (P-S6), a downstream marker of mTORC1 activity, and VGLUT2 (Fig. 4A, 4B, Extended Data Fig. 4-1). Increased levels of P-S6 in VGLUT2-positive brain regions were observed in NPRL2 nKO compared to control, suggesting a failure to repress mTORC1 signaling. We also co-stained sections with NeuN, GFAP and PS6 to determine if the effect on P-S6 was cell autonomous. We observed no increase in P-S6 staining in cells co-stained with GFAP in the NPRL2 nKO compared to control, consistent with a cell autonomous 
effect (Extended Data Fig. 4-2). Western blot analysis of protein extracts showed a significant increase in P-S6 abundance in the NPRL2 nKO compared to WT (Fig. 4C, Extended Data Fig. 4-1), consistent with histological observations. We also observed a trend of increased phosphorylation of S6-kinase, and a small reduction in total AKT and phosphorylated AKT (S473) in the NPRL2 nKO. These data suggest that the regulation of $\mathrm{mTORC1}$ through NPRL2 is necessary to prevent hyperactive mTORC1 signaling in glutamatergic neurons despite an intact TSC1/2 complex.

mTORC1 activity is implicated in diverse cellular processes that impact the regulation of metabolic homeostasis within cells. To determine if the loss of NPRL2 in glutamatergic neurons contributes to metabolic defects in the brain, metabolites were extracted and quantified using targeted small molecule mass spectrometry. NPRL2 nKO extracts showed a significant reduction in the abundance of all amino acids except glutamate, aspartate and leucine, compared to littermate controls (Fig. 5A). Remarkably, the abundance of glutamine was $60 \%$ lower in the NPRL2 nKO brain extracts, compared to littermate controls. We also observed a significant decrease in dopamine, but no change in serotonin, histamine, creatinine or taurine (Fig. 5B). The abundance of carnosine was significantly reduced, while putrescine was significantly increased in the NPRL2 nKO brain (Fig. 5B). Since increased levels of putrescine are associate with cell death, we measured the expression of genes that are transcriptionally induced by neuronal cell death, including Bax, Dapk2, Dapk3, and Pcna, but found no significant change at P16 (Extended Data Fig. 5-1) (Chiang et al., 2001; Takao et al., 2006). No significant differences were observed in the abundance of measured acylcarnitines, sphingolipids, lysophosphatidylcholines (Extended Data Fig. 5-2), nor phosphatidylcholines between NPRL2 nKO and control extracts. Collectively, these data show an important function of NPRL2 in regulating amino acid homeostasis in brain.

\section{NPRL2-dependent mTORC1 activity controls sodium channel expression}


281 The electrophysiological characteristics of the NPRL2 nKO neurons suggested a dysregulation of sodium ion channel expression could contribute to the increased amplitude of the action potential. We used quantitative RT-PCR to measure the expression of sodium channels in the brains of P16 NPRL2 nKO and littermate controls. We observed a significant upregulation of $\operatorname{Scn} 1 A, \operatorname{Scn} 1 B, \operatorname{Scn} 2 A$ and $\operatorname{Scn} 2 B$ in the NPRL2 nKO compared to control, but no change in Scn3A, Scn3B, Scn4B or Scn5A (Fig. 6A). Expression of potassium channels: Kv1.1, Kv1.3, Kv1.4 and Kv2.2, were not significantly changed; whereas Kv 1.6 was induced 1.2-fold (Extended Data Fig. 6-1). We next performed western blot analysis on membrane fractions and observed a significant increase in SCN1A protein expression in the NPRL2 nKO, compared to internal control (Fig. 6B).

We next hypothesized that mTORC1 activation contributes to the expression of sodium channels in neurons. We generated primary neuron cultures from $N p r / 2^{\operatorname{lox} P / l o x P}$ animals and infected them with either Cre-expressing lentivirus or negative control virus. Neurons were treated at DIV 7 with rapamycin, an inhibitor of mTORC1 kinase activity, and quantitative RT-PCR was used to measure the expression of $\mathrm{Nprl} 2$ and $\mathrm{Scn} 1 \mathrm{~A}$. Treatment of $\mathrm{Nprl} 2^{\operatorname{loxP} / \mathrm{loxP}}$ neurons with Cre-expressing lentivirus produced a $>90 \%$ reduction of $\mathrm{Npr} / 2$ and a significant up-regulation of $\operatorname{Scn} 1 \mathrm{~A}$, compared to control infected neurons (Fig. 6C). Moreover, rapamycin treatment blocked the upregulation of Scn1A in NPRL2 knockout neurons (Fig. 6C). These observations support an important role of NPRL2 and mTORC1 activity in the regulation of $\operatorname{Scn} 1 A$ in neurons.

\section{Discussion:}

Human mutations in NPRL2 are associated with a spectrum of neurological disorders, including autism, epilepsy, and SUDEP. Here we show that loss of NPRL2 in glutamatergic neurons causes early lethality following seizures, increases the strength of electrically stimulated action potentials, and significantly alters amino acid homeostasis in the brain. Remarkably, the onset of seizures in the NPRL2 nKO mice 
occurred shortly before the start of the light-cycle, consistent with observations of GATOR1-dependent nocturnal seizures in humans (Korenke et al., 2016; Picard et al., 2014). These circadian differences between human and mouse observations could be due to their diurnal versus nocturnal nature. While human genomic sequencing studies have identified a greater numbers of disease-linked mutations in the larger GATOR1 subunit called DEPDC5 (Baldassari et al., 2019) compared to NPRL2, our data show that strict loss-of-function mutations in NPRL2 cause early lethality and may be under-represented as a genetic risk factor for SUDEP. As previous studies have showed global deletion of NPRL2 results in embryonic lethality and defective fetal liver hematopoiesis (Dutchak et al., 2015), the mechanisms that underlie epilepsy-linked NPRL2 somatic mutations may elicit distinct changes in the GATOR1 structure that impair cell-type specific mTORC1 regulatory functions. Collectively, these data support genetic screening of GATOR1 mutations during risk assessments for SUDEP.

GATOR1 functions as an evolutionarily conserved intracellular amino acid-signaling complex that has a critical function to maintain intracellular amino acid homeostasis (Bar-Peled et al., 2013; Chen et al., 2017; Dutchak et al., 2018; Dutchak et al., 2015). Previous studies have shown that defects in NPRL2 cause significant changes in cellular glutamine and glutamate homeostasis, and alter the expression of metabolic enzymes that control these reactions (Dutchak et al., 2018). Here, we show that deletion of NPRL2 from glutamatergic neurons causes a $60 \%$ reduction in brain glutamine levels, but an insignificant decrease in glutamate levels. These data suggest that the absence of NPRL2 in glutamatergic neurons, the high demand of glutamate to support neuronal functions, including synaptic transmission and protein translation, can be met by increased metabolic supply of glutamine from astrocytes (Schousboe et al., 2014) or potential rewiring of secondary metabolic pathways (Chen et al., 2017). Since the glutamate-glutamine cycle relies on glutamine from astrocytes to support neural populations and prevent neurotransmitter depletion, these data suggest that the ability of astrocytes to regenerate glutamine is insufficient when NPRL2 is impaired in neurons (Eid et al., 2016; Schousboe et al., 2013). 
Subsequently, these metabolic deficiencies may contribute to the changes in excitatory glutamate signaling associated with seizures (Eid et al., 2016; Schousboe et al., 2014), or by substrate deficiencies in the inhibitory GABAergic system (Swaminathan et al., 2018).

We also identified other amino acids deficiencies, including arginine, which may represent homeostatic mechanism to downregulate mTORC1 activity in the brain (Chantranupong et al., 2016). We did not detect any change in creatinine or taurine in the NPRL2 nKO, indicating these secondary pathways of energy metabolism are not affected. However, the increase in putrescine indicates a neurodegenerative metabolic process may be stimulated prior to death, whereas no changes in genetic markers of apoptosis were observed in living mice at P16 (Chiang et al., 2001; Takao et al., 2006). No changes in brain acylcarnitines, sphingolipids, lysophosphatidylcholines or phosphatidylcholines were observed. Collectively, these data support NPRL2 as a specific regulator of amino acids and their metabolic pathways in the brain.

Early studies showed patients with activating mutations in mTORC1 signaling presented with focal cortical dysplasia and neurodevelopmental cortical malformations (Lim et al., 2015; Mirzaa et al., 2016), with recent evidence supporting a novel two-hit mosaic mutation mechanism as the etiology of the focal cortical dysplasia (Ribierre et al., 2018). While no gross morphological differences were apparent in the NPRL2 nKO brain, layer IV-V cortical neurons of the NPRL2 nKO mice had larger cell bodies, with reduced dendritic branching, similar to previous reports when DEPDC5 is deleted using a pan-neuronal Cre-driver (Yuskaitis et al., 2018). These data support a critical role for strict mTORC1 regulation by the GATOR1 amino acid signaling pathway in early neuronal development.

Our data show that loss of NPRL2 in glutamatergic neurons is sufficient to increase mTORC1 signal transduction, despite in an intact TSC1/2 complex (Bourdeau Julien et al., 2018). Previous studies have shown that mTORC1 is localized to the axons and dendrites of neurons (Takei et al., 2004; Terenzio et 
al., 2018), but its biological impact has not been clearly defined. Our data support an important link between hyperactive mTORC1 and changes in the electrical activity of the neurons by affecting the expression of sodium channels. Our electrophysiological analysis shows that loss of NPRL2 is sufficient to increase the strength of evoked APs, with a significantly greater amplitude and faster rise time during the depolarization current step. Consistent with these changes in electrophysiological parameters, we observed a significant upregulation of $\operatorname{Sen} 1 a, \operatorname{Sen} 1 b, \operatorname{Sen} 2 a$, and $\operatorname{Sen} 2 b$ in the brain of the NPRL2 nKO mice. Our loss-of-function studies in primary neuronal cultures also show consistent up-regulation of Scn1a expression when NPRL2 is deleted, consistent with a cell autonomous effect. Moreover, the upregulation of Scn1a expression is prevented by treatment with rapamycin, the pharmacological inhibitor of mTORC1. As genetic studies have identified more than 700 mutations in genes that code for voltage-gated sodium channels in epilepsy (Kaplan et al., 2016), and reports of GATOR1 and Scn1a mutations contributing to temporal epilepsy (Baldassari et al., 2016), our data now implicate the NPRL2 and mTORC1 regulatory pathways in controlling Scn1a expression.

Multiple mutations in several voltage-gated sodium channels have been linked to human epilepsy (Menezes et al., 2020). For example, the gain-of-function T226M mutation in Scn1a has been found in children with developmental and epileptic encephalopathy (Berecki et al., 2019) and gain-of-function mutations in Scn2a have been shown to elicit seizures, behavioral arrest, and behavioral abnormalities in mice (Kearney et al., 2001). The phenotype of our NPRL2 nKO model is consistent with the upregulation of Scn1A causing a gain-of-function effect. In contrast, loss-of-function Scn1A mutations are associated with genetic epilepsy with febrile seizures plus (GEFS+) and Dravet syndrome, consistent with decreased activity of inhibitory GABAergic neurons (Escayg and Goldin, 2010; Ruffolo et al., 2018). Future research investigating the contribution of voltage-gated sodium channels in distinct neuronal sub-types and brain regions will be of interest for targeted pharmacological therapies. 
Genomic studies have shown that $8 \%$ of patients carrying DEPDC5 mutations present with depression (Baldassari et al., 2019). Our metabolite analysis of the NPRL2 nKO brain show significantly lower levels of the neurotransmitter dopamine, which is linked to depression (Dunlop and Nemeroff, 2007). Intriguingly, animal studies have shown that dopamine levels are decreased in rat models expressing epilepsy linked Scn1a-mutations (Ohmori et al., 2014), providing a functional link between the overexpression of Scn1A and reduced dopamine levels in the Nprl2 nKO model. Collectively, these observations suggest a novel mechanism linking mTORC1 and the expression of sodium channels and the mesolimbic dopamine reward pathway.

In summary, we have shown that the NPRL2 expression in glutamatergic neurons is essential to prevent seizures prior to death at $\mathrm{P} 20$, and contributes to the regulation of amino acid homeostasis and sodium channel expression in the brain. While the impact of altered amino acid metabolism in the brain remains under investigation, our findings represent a novel link between cellular metabolic regulation and neuronal activity. We conclude that NPRL2 functions as an important regulator of mTORC1 activity that controls the expression of epilepsy-linked Scn1a expression to regulate the strength of neuronal action potentials. 


\section{References:}

Baldassari, S., Licchetta, L., Tinuper, P., Bisulli, F., and Pippucci, T. (2016). GATOR1 complex: the common genetic actor in focal epilepsies. J Med Genet 53, 503-510.

Baldassari, S., Picard, F., Verbeek, N.E., van Kempen, M., Brilstra, E.H., Lesca, G., Conti, V., Guerrini, R., Bisulli, F., Licchetta, L., et al. (2019). The landscape of epilepsy-related GATOR1 variants. Genet Med 21, 398-408.

Bar-Peled, L., Chantranupong, L., Cherniack, A.D., Chen, W.W., Ottina, K.A., Grabiner, B.C., Spear, E.D., Carter, S.L., Meyerson, M., and Sabatini, D.M. (2013). A Tumor suppressor complex with GAP activity for the Rag GTPases that signal amino acid sufficiency to mTORC1. Science 340, 1100-1106.

Berecki, G., Bryson, A., Terhag, J., Maljevic, S., Gazina, E.V., Hill, S.L., and Petrou, S. (2019). SCN1A gain of function in early infantile encephalopathy. Ann Neurol 85, 514-525.

Bourdeau Julien, I., Sephton, C.F., and Dutchak, P.A. (2018). Metabolic Networks Influencing Skeletal Muscle Fiber Composition. Front Cell Dev Biol 6, 125.

Chantranupong, L., Scaria, S.M., Saxton, R.A., Gygi, M.P., Shen, K., Wyant, G.A., Wang, T., Harper, J.W., Gygi, S.P., and Sabatini, D.M. (2016). The CASTOR Proteins Are Arginine Sensors for the mTORC1 Pathway. Cell 165, 153-164.

Chen, J., Sutter, B.M., Shi, L., and Tu, B.P. (2017). GATOR1 regulates nitrogenic cataplerotic reactions of the mitochondrial TCA cycle. Nat Chem Biol 13, 1179-1186.

Chiang, L.W., Grenier, J.M., Ettwiller, L., Jenkins, L.P., Ficenec, D., Martin, J., Jin, F., DiStefano, P.S., and Wood, A. (2001). An orchestrated gene expression component of neuronal programmed cell death revealed by cDNA array analysis. Proc Natl Acad Sci U S A 98, 2814-2819.

Curatolo, P., Moavero, R., and de Vries, P.J. (2015). Neurological and neuropsychiatric aspects of tuberous sclerosis complex. Lancet Neurol 14, 733-745.

Dibbens, L.M., de Vries, B., Donatello, S., Heron, S.E., Hodgson, B.L., Chintawar, S., Crompton, D.E., Hughes, J.N., Bellows, S.T., Klein, K.M., et al. (2013). Mutations in DEPDC5 cause familial focal epilepsy with variable foci. Nat Genet 45, 546-551.

Dunlop, B.W., and Nemeroff, C.B. (2007). The role of dopamine in the pathophysiology of depression. Arch Gen Psychiatry 64, 327-337.

Dutchak, P.A., Estill-Terpack, S.J., Plec, A.A., Zhao, X., Yang, C., Chen, J., Ko, B., Deberardinis, R.J., Yu, Y., and Tu, B.P. (2018). Loss of a Negative Regulator of mTORC1 Induces Aerobic Glycolysis and Altered Fiber Composition in Skeletal Muscle. Cell Rep 23, 1907-1914.

Dutchak, P.A., Laxman, S., Estill, S.J., Wang, C., Wang, Y., Wang, Y., Bulut, G.B., Gao, J., Huang, L.J., and Tu, B.P. (2015). Regulation of Hematopoiesis and Methionine Homeostasis by mTORC1 Inhibitor NPRL2. Cell Rep 12, 371-379.

Efeyan, A., Zoncu, R., Chang, S., Gumper, I., Snitkin, H., Wolfson, R.L., Kirak, O., Sabatini, D.D., and Sabatini, D.M. (2013). Regulation of mTORC1 by the Rag GTPases is necessary for neonatal autophagy and survival. Nature 493, 679-683. 
Eid, T., Gruenbaum, S.E., Dhaher, R., Lee, T.-S.W., Zhou, Y., and Danbolt, N.C. (2016). The GlutamateGlutamine Cycle in Epilepsy. In The Glutamate/GABA-Glutamine Cycle: Amino Acid Neurotransmitter Homeostasis, A. Schousboe, and U. Sonnewald, eds. (Cham: Springer International Publishing), pp. 351400.

Escayg, A., and Goldin, A.L. (2010). Sodium channel SCN1A and epilepsy: mutations and mechanisms. Epilepsia 51, 1650-1658.

Griffith, J.L., and Wong, M. (2018). The mTOR pathway in treatment of epilepsy: a clinical update. Future Neurol 13, 49-58.

Kaplan, D.I., Isom, L.L., and Petrou, S. (2016). Role of Sodium Channels in Epilepsy. Cold Spring Harb Perspect Med 6.

Kearney, J.A., Plummer, N.W., Smith, M.R., Kapur, J., Cummins, T.R., Waxman, S.G., Goldin, A.L., and Meisler, M.H. (2001). A gain-of-function mutation in the sodium channel gene Scn2a results in seizures and behavioral abnormalities. Neuroscience 102, 307-317.

Korenke, G.C., Eggert, M., Thiele, H., Nurnberg, P., Sander, T., and Steinlein, O.K. (2016). Nocturnal frontal lobe epilepsy caused by a mutation in the GATOR1 complex gene NPRL3. Epilepsia 57, e60-63.

Lakhan, R., Kumari, R., Misra, U.K., Kalita, J., Pradhan, S., and Mittal, B. (2009). Differential role of sodium channels SCN1A and SCN2A gene polymorphisms with epilepsy and multiple drug resistance in the north Indian population. Br J Clin Pharmacol 68, 214-220.

Laplante, M., and Sabatini, D.M. (2009). mTOR signaling at a glance. J Cell Sci 122, 3589-3594.

Lim, J.S., Kim, W.I., Kang, H.C., Kim, S.H., Park, A.H., Park, E.K., Cho, Y.W., Kim, S., Kim, H.M., Kim, J.A., et al. (2015). Brain somatic mutations in MTOR cause focal cortical dysplasia type II leading to intractable epilepsy. Nat Med 21, 395-400.

Liu, G.Y., and Sabatini, D.M. (2020). mTOR at the nexus of nutrition, growth, ageing and disease. Nature Reviews Molecular Cell Biology 21, 183-203.

Meikle, L., Talos, D.M., Onda, H., Pollizzi, K., Rotenberg, A., Sahin, M., Jensen, F.E., and Kwiatkowski, D.J. (2007). A mouse model of tuberous sclerosis: neuronal loss of Tsc1 causes dysplastic and ectopic neurons, reduced myelination, seizure activity, and limited survival. J Neurosci 27, 5546-5558.

Menezes, L.F.S., Sabia Junior, E.F., Tibery, D.V., Carneiro, L.D.A., and Schwartz, E.F. (2020). EpilepsyRelated Voltage-Gated Sodium Channelopathies: A Review. Front Pharmacol 11, 1276.

Mirzaa, G.M., Campbell, C.D., Solovieff, N., Goold, C., Jansen, L.A., Menon, S., Timms, A.E., Conti, V., Biag, J.D., Adams, C., et al. (2016). Association of MTOR Mutations With Developmental Brain Disorders, Including Megalencephaly, Focal Cortical Dysplasia, and Pigmentary Mosaicism. JAMA Neurol 73, 836845.

Nascimento, F.A., Borlot, F., Cossette, P., Minassian, B.A., and Andrade, D.M. (2015). Two definite cases of sudden unexpected death in epilepsy in a family with a DEPDC5 mutation. Neurol Genet 1, e28.

Nault, F., and De Koninck, P. (2010). Dissociated Hippocampal Cultures. In Protocols for Neural Cell Culture: Fourth Edition, L.C. Doering, ed. (Totowa, NJ: Humana Press), pp. 137-159. 
Ohmori, I., Kawakami, N., Liu, S., Wang, H., Miyazaki, I., Asanuma, M., Michiue, H., Matsui, H., Mashimo, T., and Ouchida, M. (2014). Methylphenidate improves learning impairments and hyperthermia-induced seizures caused by an Scn1a mutation. Epilepsia 55, 1558-1567.

Picard, F., Makrythanasis, P., Navarro, V., Ishida, S., de Bellescize, J., Ville, D., Weckhuysen, S., Fosselle, E., Suls, A., De Jonghe, P., et al. (2014). DEPDC5 mutations in families presenting as autosomal dominant nocturnal frontal lobe epilepsy. Neurology 82, 2101-2106.

Ribierre, T., Deleuze, C., Bacq, A., Baldassari, S., Marsan, E., Chipaux, M., Muraca, G., Roussel, D., Navarro, V., Leguern, E., et al. (2018). Second-hit mosaic mutation in mTORC1 repressor DEPDC5 causes focal cortical dysplasia-associated epilepsy. J Clin Invest 128, 2452-2458.

Ricos, M.G., Hodgson, B.L., Pippucci, T., Saidin, A., Ong, Y.S., Heron, S.E., Licchetta, L., Bisulli, F., Bayly, M.A., Hughes, J., et al. (2016). Mutations in the mammalian target of rapamycin pathway regulators NPRL2 and NPRL3 cause focal epilepsy. Ann Neurol 79, 120-131.

Ruffolo, G., Cifelli, P., Roseti, C., Thom, M., van Vliet, E.A., Limatola, C., Aronica, E., and Palma, E. (2018). A novel GABAergic dysfunction in human Dravet syndrome. Epilepsia 59, 2106-2117.

Sancak, Y., Bar-Peled, L., Zoncu, R., Markhard, A.L., Nada, S., and Sabatini, D.M. (2010). Ragulator-Rag complex targets mTORC1 to the lysosomal surface and is necessary for its activation by amino acids. Cell 141, 290-303.

Schousboe, A., Bak, L.K., and Waagepetersen, H.S. (2013). Astrocytic Control of Biosynthesis and Turnover of the Neurotransmitters Glutamate and GABA. Front Endocrinol (Lausanne) 4, 102.

Schousboe, A., Scafidi, S., Bak, L.K., Waagepetersen, H.S., and McKenna, M.C. (2014). Glutamate metabolism in the brain focusing on astrocytes. Adv Neurobiol 11, 13-30.

Sephton, C.F., Tang, A.A., Kulkarni, A., West, J., Brooks, M., Stubblefield, J.J., Liu, Y., Zhang, M.Q., Green, C.B., Huber, K.M., et al. (2014). Activity-dependent FUS dysregulation disrupts synaptic homeostasis. Proc Natl Acad Sci U S A 111, E4769-4778.

Shen, K., Valenstein, M.L., Gu, X., and Sabatini, D.M. (2019). Arg-78 of Nprl2 catalyzes GATOR1stimulated GTP hydrolysis by the Rag GTPases. J Biol Chem 294, 2970-2975.

Specchio, N., Pietrafusa, N., Trivisano, M., Moavero, R., De Palma, L., Ferretti, A., Vigevano, F., and Curatolo, P. (2020). Autism and Epilepsy in Patients With Tuberous Sclerosis Complex. Front Neurol 11, 639.

Swaminathan, A., Hassan-Abdi, R., Renault, S., Siekierska, A., Riche, R., Liao, M., de Witte, P.A.M., Yanicostas, C., Soussi-Yanicostas, N., Drapeau, P., et al. (2018). Non-canonical mTOR-Independent Role of DEPDC5 in Regulating GABAergic Network Development. Curr Biol 28, 1924-1937 e1925.

Takao, K., Rickhag, M., Hegardt, C., Oredsson, S., and Persson, L. (2006). Induction of apoptotic cell death by putrescine. Int J Biochem Cell Biol 38, 621-628.

Takei, N., Inamura, N., Kawamura, M., Namba, H., Hara, K., Yonezawa, K., and Nawa, H. (2004). Brainderived neurotrophic factor induces mammalian target of rapamycin-dependent local activation of translation machinery and protein synthesis in neuronal dendrites. J Neurosci 24, 9760-9769. 


\section{9}

Talos, D.M., Sun, H., Zhou, X., Fitzgerald, E.C., Jackson, M.C., Klein, P.M., Lan, V.J., Joseph, A., and Jensen, F.E. (2012). The interaction between early life epilepsy and autistic-like behavioral consequences: a role for the mammalian target of rapamycin (mTOR) pathway. PLoS One 7, e35885.

Terenzio, M., Koley, S., Samra, N., Rishal, I., Zhao, Q., Sahoo, P.K., Urisman, A., Marvaldi, L., Oses-Prieto, J.A., Forester, C., et al. (2018). Locally translated mTOR controls axonal local translation in nerve injury. Science 359, 1416-1421.

Vong, L., Ye, C., Yang, Z., Choi, B., Chua, S., Jr., and Lowell, B.B. (2011). Leptin action on GABAergic neurons prevents obesity and reduces inhibitory tone to POMC neurons. Neuron 71, 142-154.

Weckhuysen, S., Marsan, E., Lambrecq, V., Marchal, C., Morin-Brureau, M., An-Gourfinkel, I., Baulac, M., Fohlen, M., Kallay Zetchi, C., Seeck, M., et al. (2016). Involvement of GATOR complex genes in familial focal epilepsies and focal cortical dysplasia. Epilepsia 57, 994-1003.

Yuskaitis, C.J., Jones, B.M., Wolfson, R.L., Super, C.E., Dhamne, S.C., Rotenberg, A., Sabatini, D.M., Sahin, M., and Poduri, A. (2018). A mouse model of DEPDC5-related epilepsy: Neuronal loss of Depdc5 causes dysplastic and ectopic neurons, increased mTOR signaling, and seizure susceptibility. Neurobiol Dis 111 , 91-101.

Zhou, Y., and Danbolt, N.C. (2014). Glutamate as a neurotransmitter in the healthy brain. J Neural Transm (Vienna) 121, 799-817. 


\section{Figure Legends:}

Figure 1. Loss of NPRL2 expression in glutamatergic neurons causes early lethality. A) Western blot analysis of NPRL2, NPRL3, DEPDC5 and GAPDH from brain protein extracts ( $n=3$ per group). B) Quantification of Western blot signal intensities relative to GAPDH ( $n=6$ per genotype). Error bars represent the mean \pm SD. C) Survival curve of NPRL2 nKO and wild-type littermate controls ( $n=12 / 40$, respectively). All NPRL2 nKO animals die 20 days after birth. D) Body mass of NPRL2 nKO and littermate controls (male $n=7 / 16$ and female $n=7 / 6$, respectively). (Extended Data Fig. 1-1). Error bars represent the mean \pm SEM. $a, p<0.05 ; c, p<0.001$.

Figure 2. NPRL2 regulates dendritic branching of cortical neurons. A) Representative images of Neurolucida tracing of the dendrites of layer IV-V cortical neurons in NPRL2 nKO and littermate controls ( $n=30$ neurons/3 mice per genotype). B) Sholl analysis show a reduced number of intersections, and C) reduced cumulative area of dendrites in the NPRL2 nKO compared to littermate controls at P16. D) Soma volume of NPRL2 nKO versus littermate controls. Scale bar $=50 \mu \mathrm{m} . \quad a, p<0.05 ; c, p<0.001$.

Figure 3. Electrophysiological properties of NPRL2 nKO neurons. A) Representative tracings of the response samples of control (black) or NPRL2 nKO (red) neurons and protocol of injected depolarization steps. B) The number of action potentials elicited by different current steps and their C) frequency. D) Example trace of the first action potential from control (black) or NPRL2 nKO (red). First action potential E) amplitude, F) rise time and G) decay slope evoked by different depolarizing steps. H) Resting membrane potential, I) rheobase current, and J) capacitance measurements from NPRL2 nKO and controls. NPRL2 nKO=23 neurons/3 mice; Control=25 neurons/3 mice. $a, \mathrm{p}<0.05 ; b, \mathrm{p}<0.01$.

Figure 4. Loss of NPRL2 increases mTORC1 signaling. Representative image of immunohistochemical staining of P-S6 (Ser240/244) and VGLUT2 in the A) hippocampus and B) cortex of NPRL2 nKO and 
control at P16. C) Western blot analysis of pS6, S6, pS6K1, S6K1, pAKT, AKT and GAPDH from soluble protein extracts $(n=3 / 3)$. (Extended Data Fig. 4-1, 4-2)

Figure 5. Metabolite analysis of P16 brain extracts. Relative abundance of A) amino acids, and B) biogenic amines were measured from NPRL2 nKO (white bars) and littermate (black bars) controls using LC-MS/MS. ( $n=7 / 10$, respectively). Error bars represent the mean \pm SEM. (Extended Data Fig. 5-1, 5-2) $a, \mathrm{p}<0.05 ; b, \mathrm{p}<0.01 ; c, \mathrm{p}<0.001$.

Figure 6. NPRL2 contributes to the regulation of sodium channel expression. A) Quantitative RT-PCR analysis of sodium voltage-gated channel alpha subunits $(\operatorname{Scn} 1 A, \operatorname{Scn} 2 A, \operatorname{Scn} 3 A$, and $\operatorname{Scn} 5 A)$ and sodium voltage-gated channel beta subunits $(S \operatorname{cn} 1 B, S \sin 2 B, S \sin 3 B$ and $S \operatorname{cn} 4 B)$ in control and NPRL2 nKO brain at P16. ( $n=5 / 4$, respectively) B) Western blot analysis of SCN1A and metabotropic glutamate receptor 1 (mGluR1) from membrane fractions of P16 WT and NPRL2 nKO brain ( $n=3 / 3)$. C) Quantitative RT-PCR analysis of $\mathrm{Npr} / 2$ and $\mathrm{Scn} 1 \mathrm{~A}$ from $\mathrm{Nprl} 2^{\text {loxP/loxP }}$ primary neurons infected with control or CRE-expressing lentivirus and treated with $10 \mathrm{nM}$ rapamycin, or DMSO control, for 24 hours (n=6/6; 2-independent experiments). (Extended Data Fig. 6-1) $a, \mathrm{p}<0.05 ; b, \mathrm{p}<0.01 ; c, \mathrm{p}<0.001$.

\section{Extended Data Figure Legends:}

Extended Data Figure 1-1. Seizure analysis of NPRL2 nKO mice. Quantification of the tonic and tonicclonic seizures of the NPRL2 nKO mice before their death.

Extended Data Figure 4-1. Quantification of A) P-S6 signal intensity from cortical immunohistochemistry staining and B) relative signaling intensity of P-S6:S6 of western blot analysis.

Extended Data Figure 4-2. Representative image of immunohistochemical staining of P-S6, NeuN, and GFAP in the cortex of A) control or B) NPRL2 nKO at P16 ( $n=3$ per genotype). Scale bar is $25 \mu \mathrm{m}$. 
564 Extended Data Figure 5-1. Quantitative gene expression of Bax, Dapk2, Dapk3, and Pcna, in control of 565 NPRL2 nKO brain. ( $n=5 / 4$ per genotype, respectively).

566 Extended Data Figure 5-2. Relative abundance of A) acylcarnitines, B) sphingolipids, and C)

567 lysophosphatidylcholines. ( $n=7 / 10$, respectively). Error bars represent the mean \pm SEM.

568 Extended Data Figure 6-1. Regulation of potassium channel expression. A) Quantitative RT-PCR analysis

569 of potassium channels (Kv1.1, Kv1.3, Kv1.4, Kv1.6, Kv2.2) in control and NPRL2 nKO brain at P16. ( $\mathrm{n}=5 / 4$,

570 respectively). $b, \mathrm{p}<0.01$

571 
A

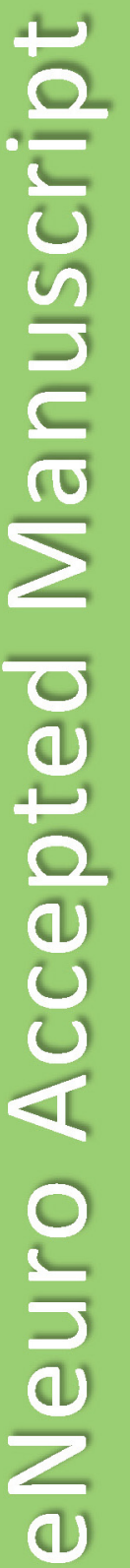

C
B
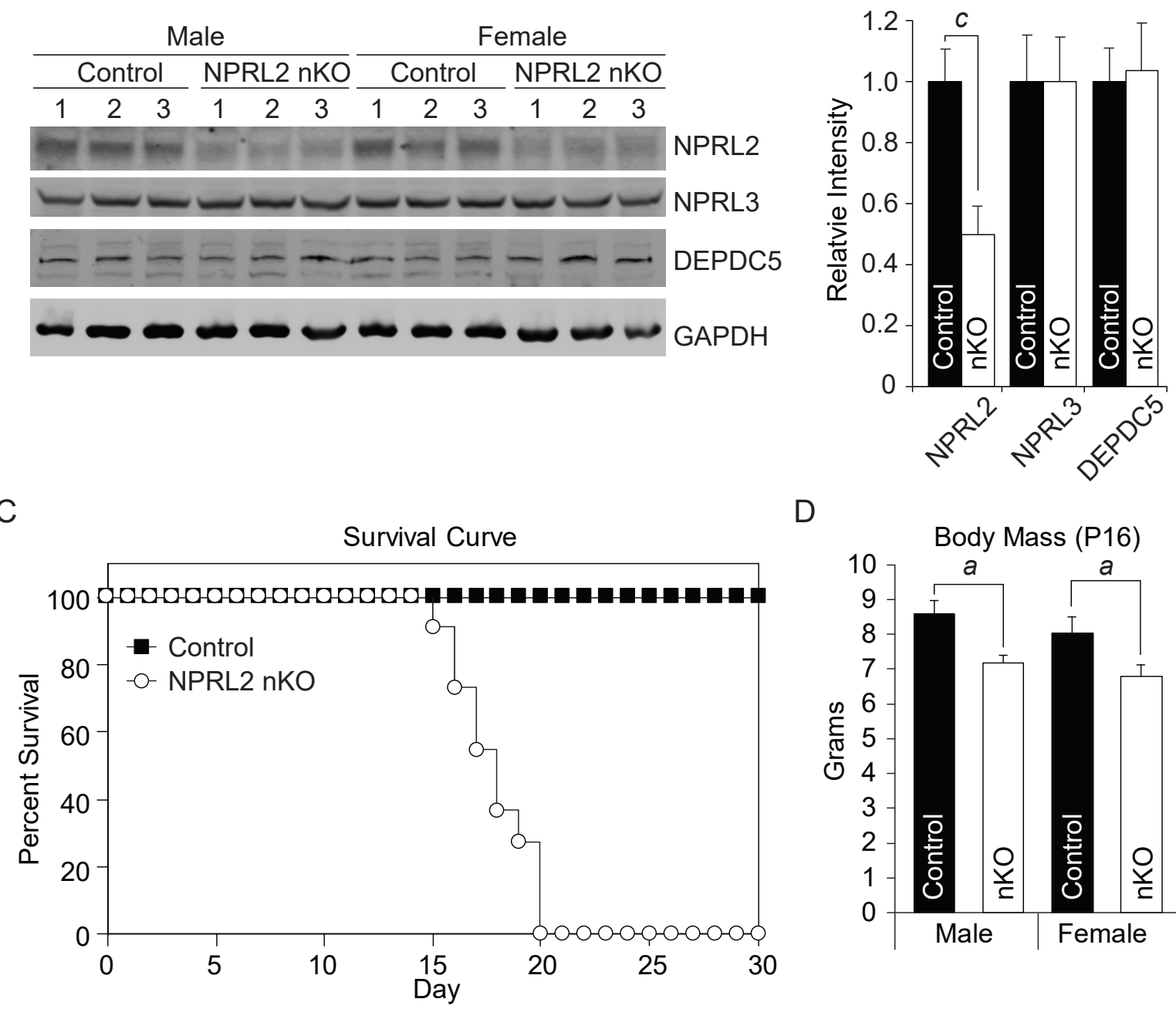

D

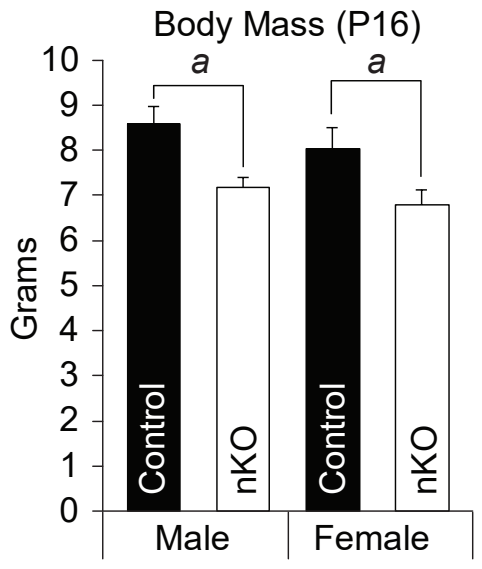


A

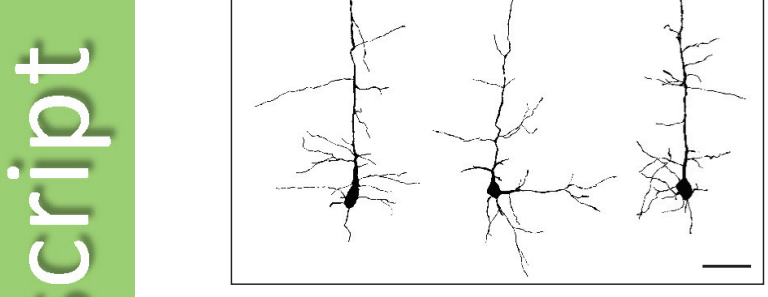

जิ

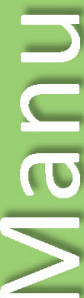

())

(U)

느)

(2)

(d)

(u)

(U)

$<$

(อ)

는

(d)

(d)
B
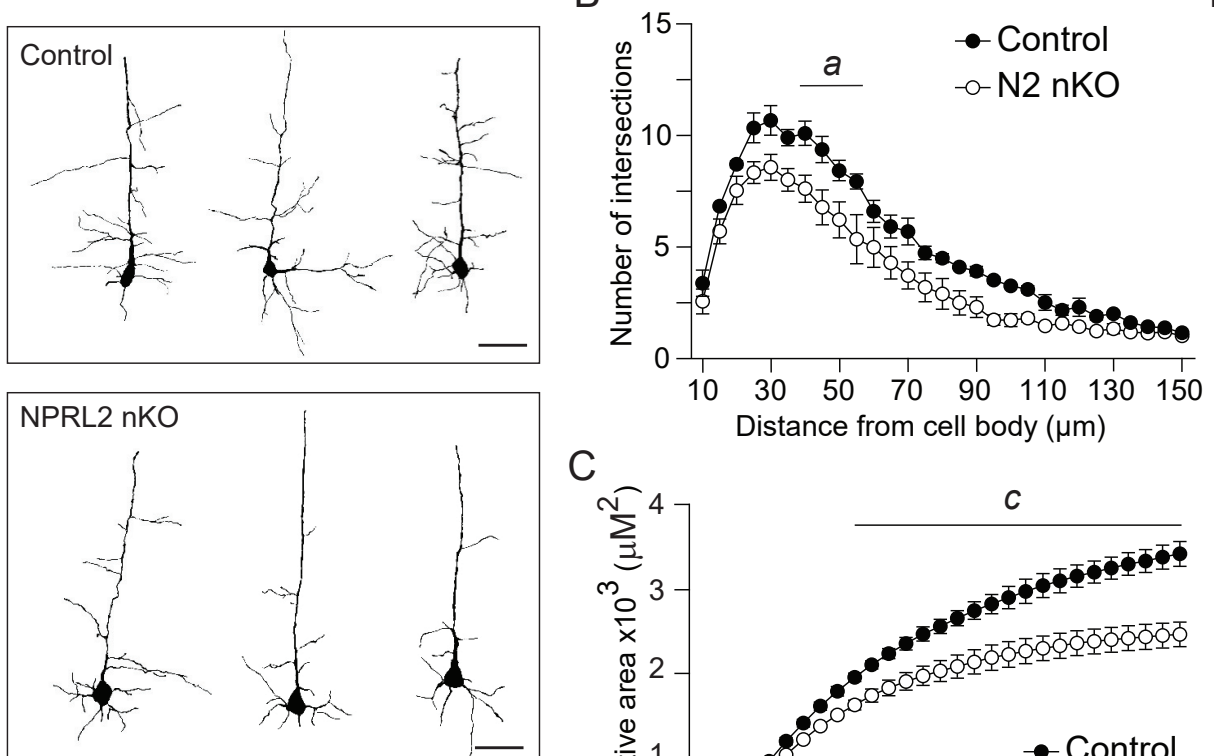

C

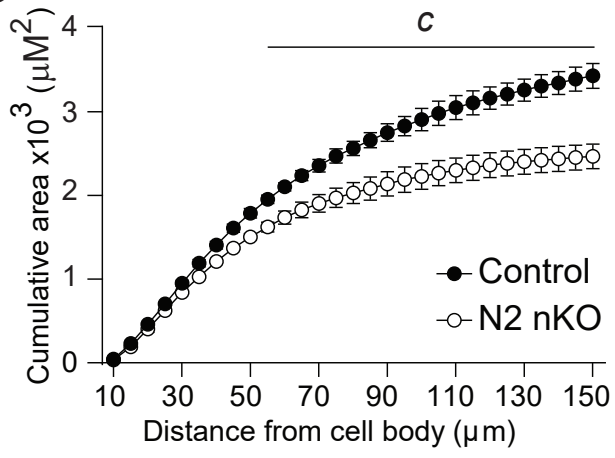

D

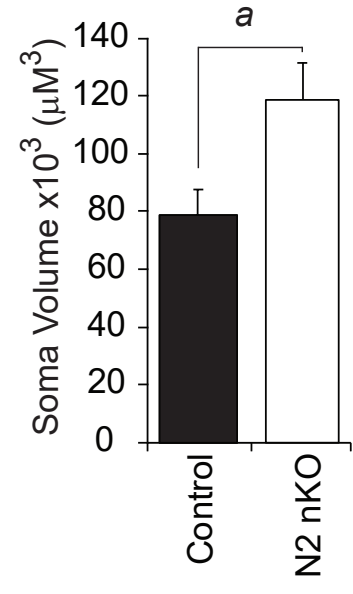




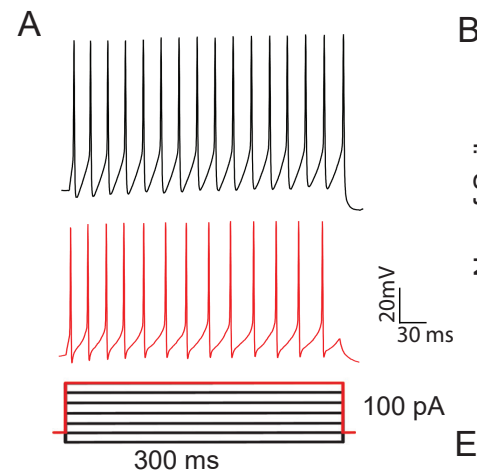

D

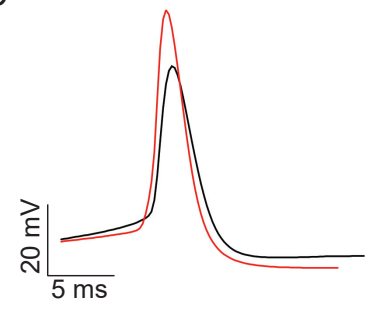

G

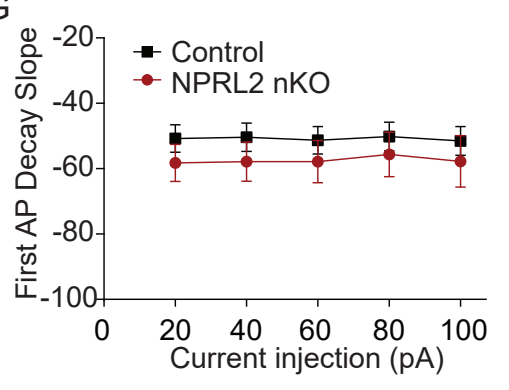

B

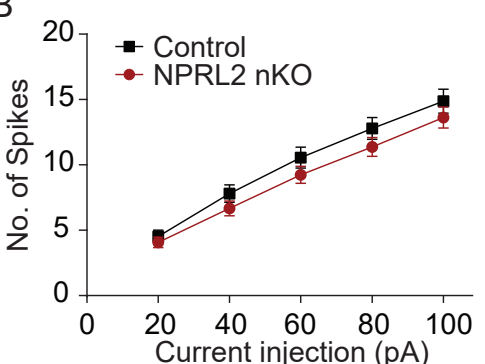

E

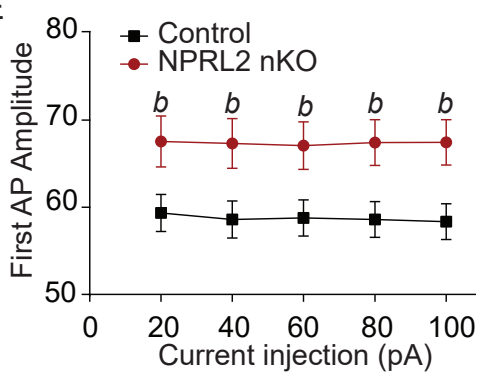

$\mathrm{H}$

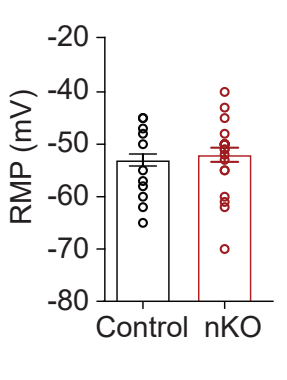

C

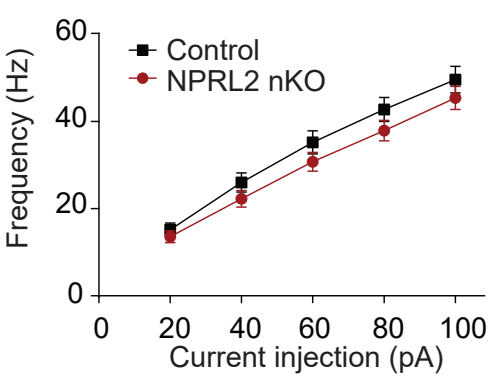

F

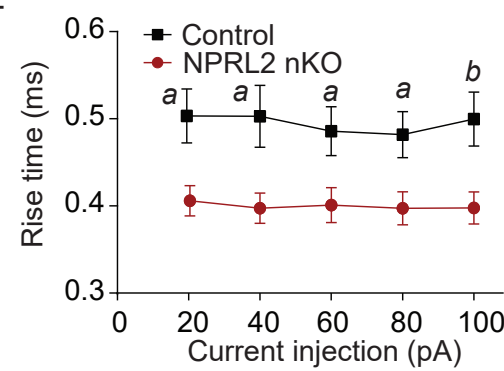

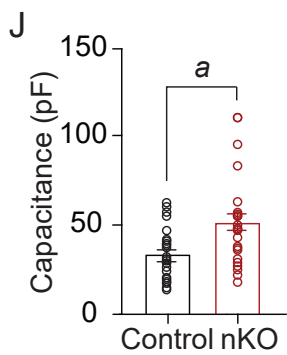




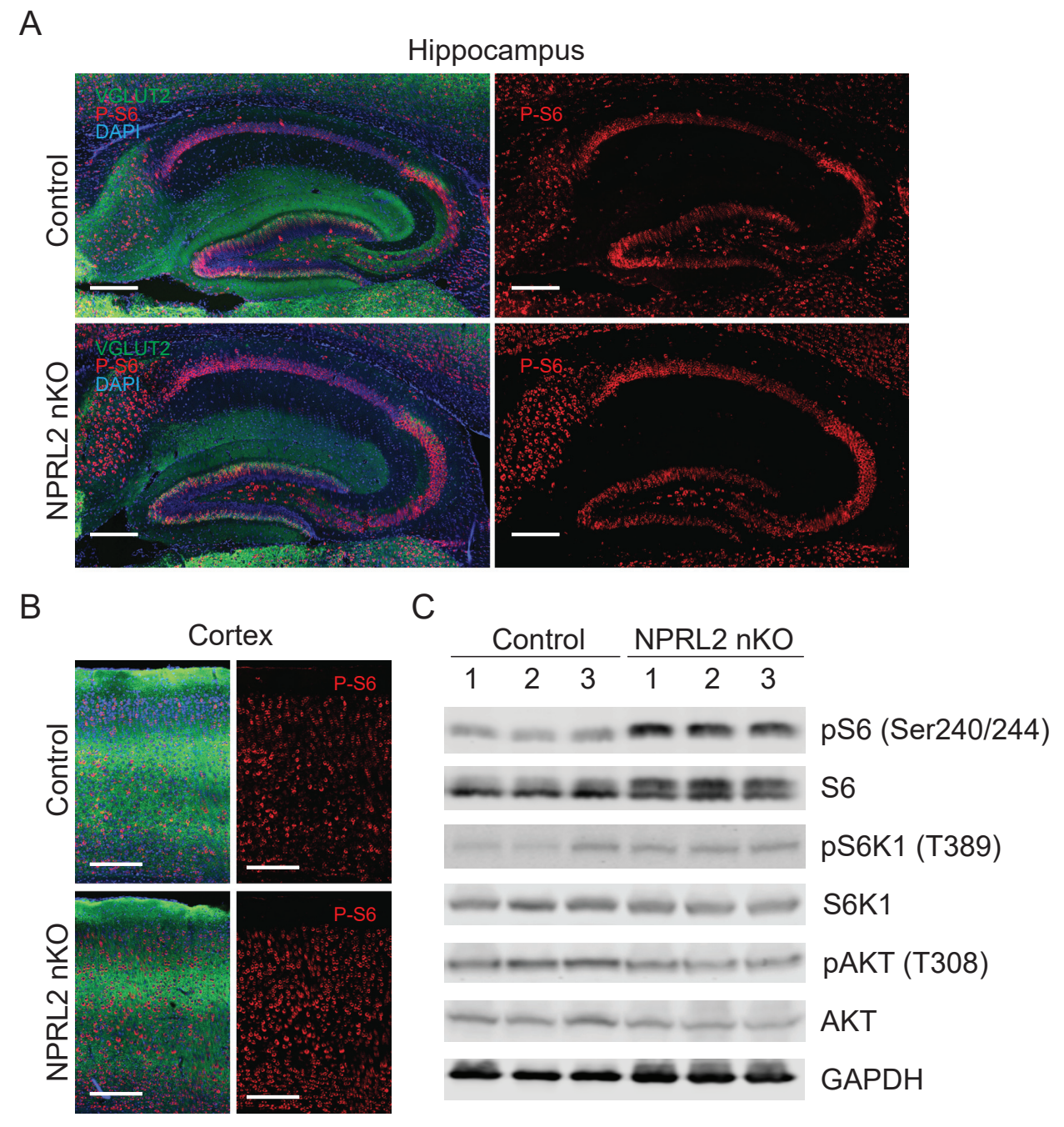




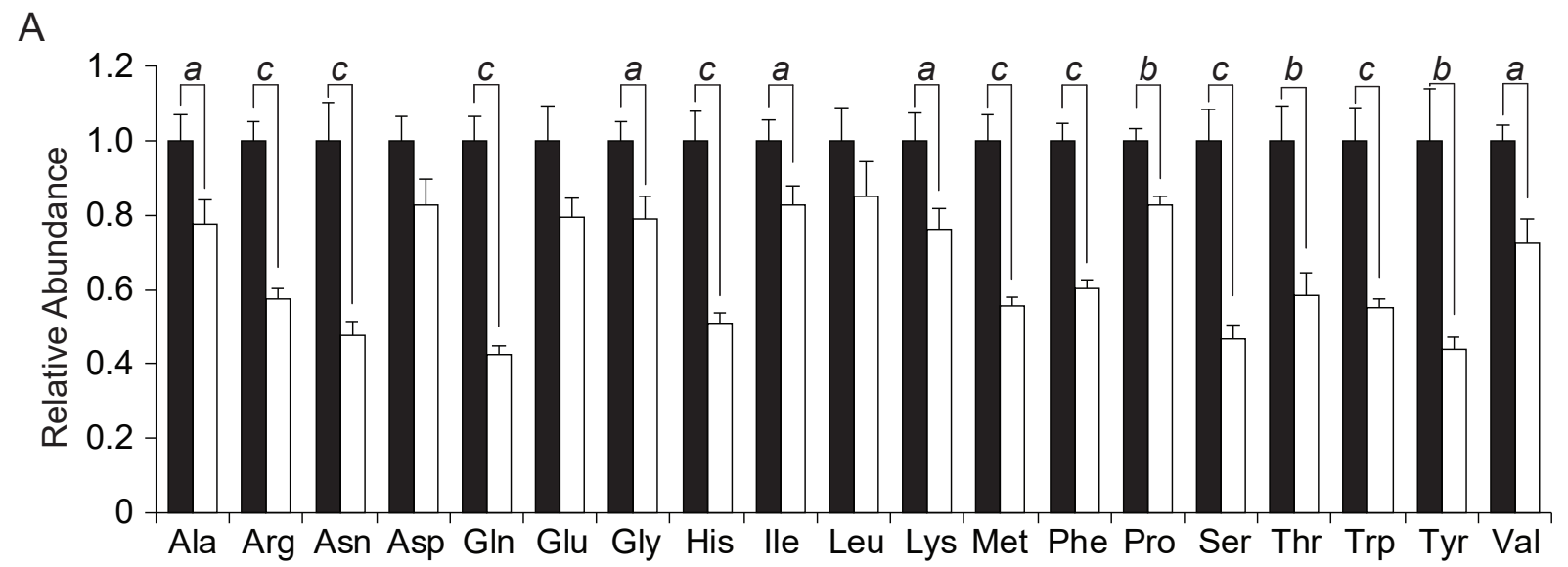

B

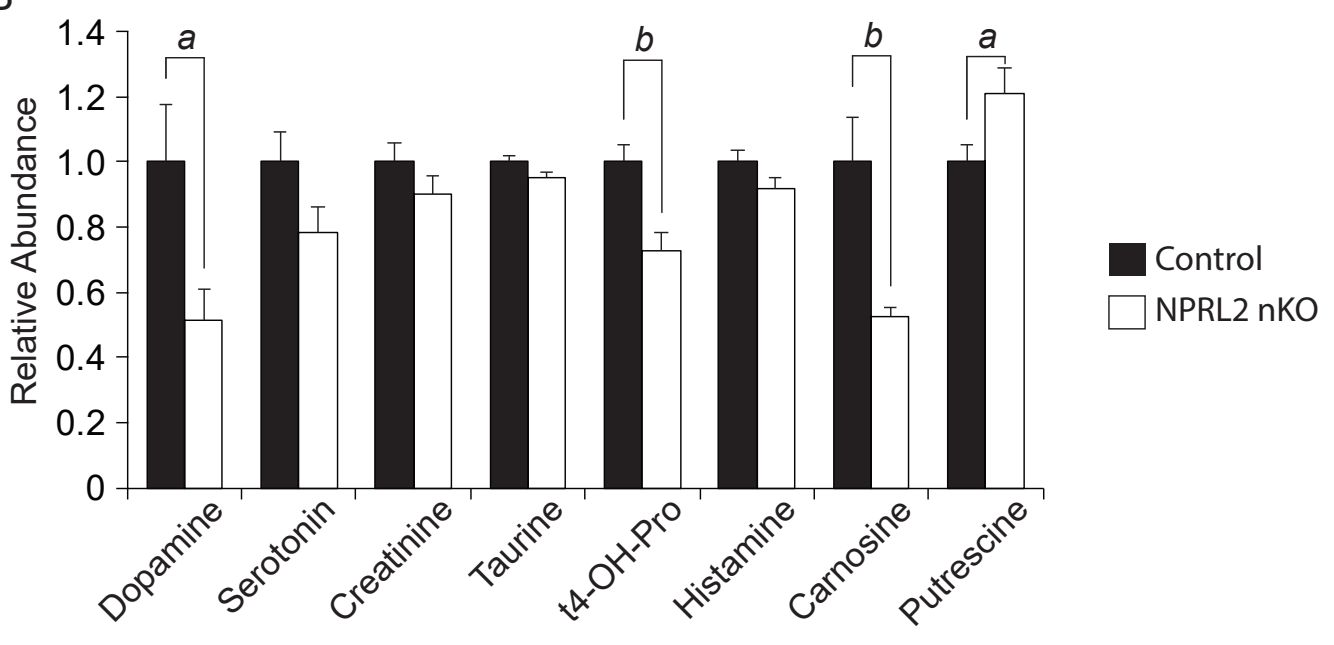


A

In vivo:

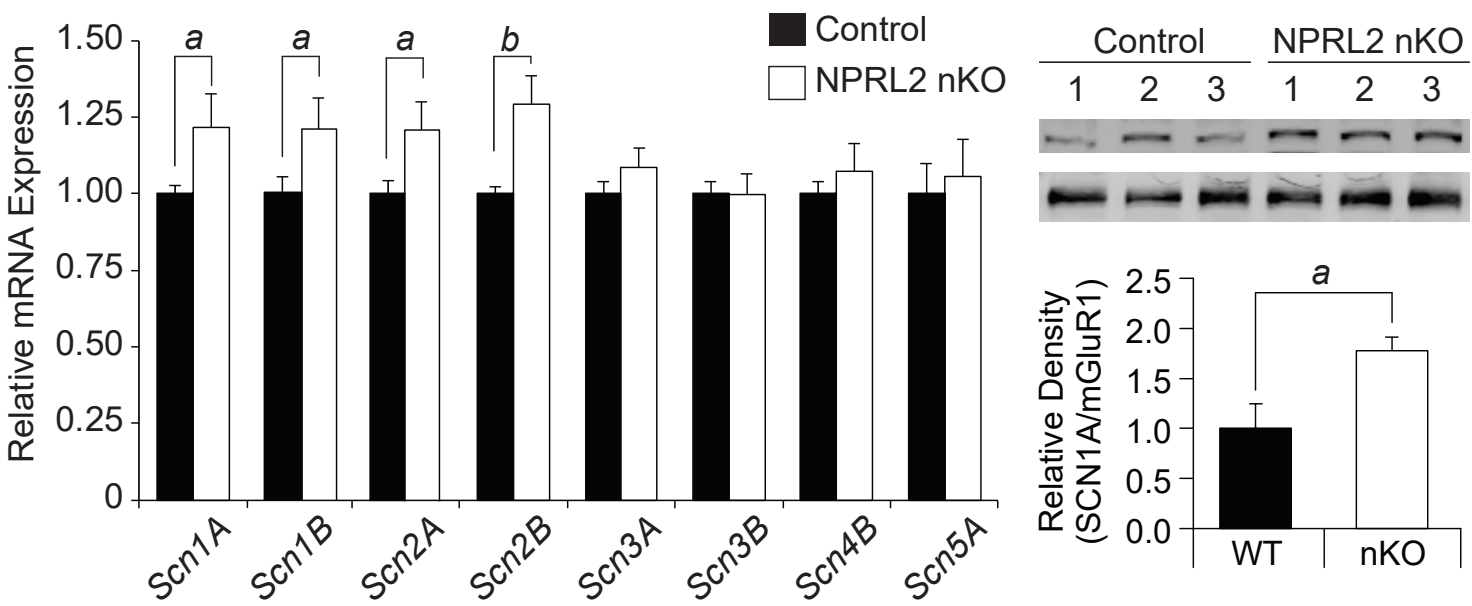

B

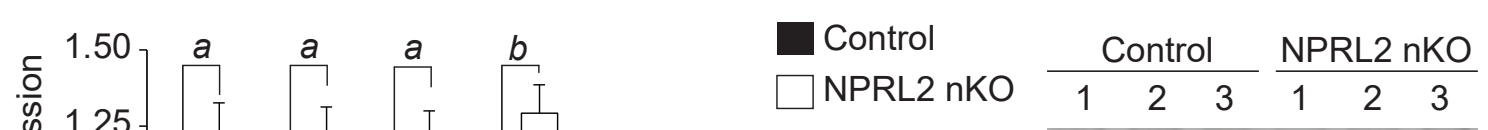

C

In vitro:
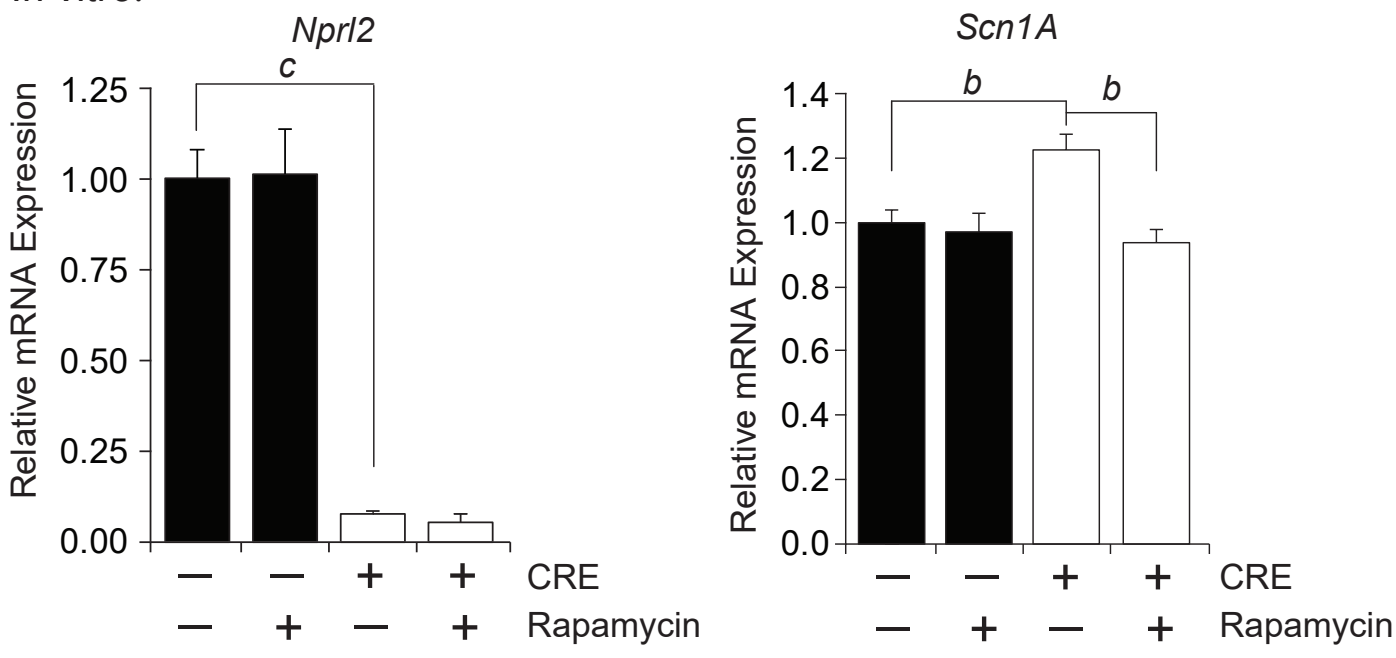


\begin{tabular}{|l|l|l|}
\hline Protein Target & Vendor & Catalog \# \\
\hline AKT & Cell Signaling Technology & 9272 \\
\hline DEPDC5 & Santa Cruz Biotechnology, Inc. & sc-86115 \\
\hline GAPDH & Sigma & G9295 \\
\hline GFAP & Millipore & MAB5541 \\
\hline mGluR1 & Fisher Scientific & 12370 \\
\hline NeuN & Millipore & AB5541 \\
\hline NPRL2 & Santa Cruz Biotechnology, Inc. & sc-376986 \\
\hline NPRL3 & Abcam & ab121346 \\
\hline p70 S6 Kinase & Cell Signaling Technology & 9202 \\
\hline Phospho-p70 S6 Kinase (T389) & Cell Signaling Technology & 9205 \\
\hline Phospho-AKT (Ser473) & Cell Signaling Technology & 9271 \\
\hline Phospho-rpS6 (Ser240/244) & Cell Signaling Technology & 2215 \\
\hline rpS6 & Cell Signaling Technology & 2217 \\
\hline Scn1A & Cell Signaling Technology & 18339 \\
\hline VGLUT2 & Synaptic Systems & 135404 \\
\hline
\end{tabular}

Table 1. List of antibodies used in this study. 


\begin{tabular}{|l|l|l|}
\hline Gene & Forward & Reverse \\
\hline Bax & GTCTCCGGCGAATTGGA & TCCACGTCAGCAATCATCCT \\
\hline Dapk2 & GCAGGGAGCAGAGCAGAG & CCTGGACCATACAATCGGCG \\
\hline Dapk3 & CGTAGATGCCGTCTCAGGAT & TGGGGACACAGGAGCGTC \\
\hline$K v 1.1$ & CCCGCCAGCGTCCATC & CTGCAGCCCTTCTAGGACAC \\
\hline$K v 1.3$ & CTTCTGGTGCGGTTCTTTGC & TACCTTGTCGTTCAGCCAGC \\
\hline$K v 1.4$ & GGCTTAACAAGTGATCGCTGC & CCAAGGTAGACCCCGGAGAT \\
\hline$K v 1.6$ & GACTGCCAATTTCTGCTTGGG & AGTTATTCAGTGGGGAGGCG \\
\hline$K v 2.2$ & AACGTAGGGGGCCTTAACCA & TTGTAGTCATCGCACACCTCC \\
\hline Pcna & CGAAGGCTTCGACACATACC & GGACATGCTGGTGAGGTTCA \\
\hline Scn1A & CTGGCTGGACTTCACTGTCAT & GATGGTCTTCAGGCCTGGAA \\
\hline Scn1B & AACACCAGCGTCGTCAAGAA & CCATCTCTGCCACAAGCCAT \\
\hline Scn2A & ACCTTTGCGTATGTAACAGAATTT & ACAGCTTCTTCACCGACTGG \\
\hline Scn2B & CGCCTAACGTCACAGTCTCT & GGACACTAAGAGTGGTGGGC \\
\hline Scn3A & GGACGTGGGGTCTGAGAATG & GTGGTACCGTTACTGTTGCG \\
\hline Scn3B & GCTCAGGAAAATGCGTCTGACTAC & TGCCTGTACATCACCTCAAGT \\
\hline Scn4B & GTGGACAACACGGTGACTCT & CACTCCTTCTTCTTCTCTCGGG \\
\hline Scn5A & AAGCTAGGCAATTTGTCGGC & GTCTTCAGGCCTGGAATAACTG \\
\hline U36B4 & CGTCCTCGTTGGAGTGACA & CGGTGCGTCAGGGATTG \\
\hline
\end{tabular}

Table 2. List of all qRT-PCR primers used in this study. 